\title{
ON THE STABLE CROSSING NUMBER OF CUBES
}

\author{
PAUL C. KAINEN
}

\begin{abstract}
Very few results are known which yield the crossing number of an infinite class of graphs on some surface. In this paper it is shown that by taking the class of graphs to be $d$-dimensional cubes $Q(d)$ and by allowing the genus of the surface to vary, we obtain upper and lower bounds on the crossing numbers which are independent of $d$. Specifically, if the genus of the surface is always $\gamma(Q(d))-k$, where $\gamma(Q(d))$ is the genus of $Q(d)$ and $k$ is a fixed nonnegative integer, then $4 k \leqq \mathrm{cr}_{\gamma(Q(d))-k}(Q(d)) \leqq 8 k$ provided that $k$ is not too large compared to $d$.
\end{abstract}

0 . Introduction. In this paper, we shall deal only with finite graphs without loops or parallel edges. If $G$ is a graph, we denote by $V(G)$ its set of vertices and $E(G)$ its set of edges. By linear algebra, we can realize any graph $G$ as a subset of three-dimensional euclidean space-simply put the set $V(G)$ of vertices in general position (no 3 collinear, no 4 coplanar) and put in the edges of $E(G)$ as straight line segments. Obviously, any two such realizations, with the subspace topologies, are homeomorphic.

By the surface $\Sigma_{g}$ of genus $g, g \geqq 0$, we mean the closed orientable 2manifold obtained by attaching $g$ handles to the sphere-e.g., $\Sigma_{1}=$ torus. An immersion $\varphi$ of $G$ in $\Sigma_{g}$ is a continuous map $\varphi: G \rightarrow \Sigma_{g}$ which is an imbedding restricted to $\varphi^{-1}(\varphi(V(G)))$ and such that, for all $y \in \Sigma_{g}$, the set $\varphi^{-1}(y)$ contains at most two points. If an immersion is $1-1$, we call it an imbedding of the graph. The image of $\varphi$, im $\varphi$ or $\varphi(G)$, is just $\left\{y \in \Sigma_{g} \mid y=\right.$ $\varphi(x)$ for some $x \in G\}$. This amounts to drawing the graph on the surface so that all vertices are distinct, two edges which intersect each other meet at either a common endpoint or at a point on the interior of both edges, and at most two edges intersect at any interior point.

For technical reasons, we shall prefer to deal only with piecewise-linear (PL) immersions. Recall that a map $f:|K| \rightarrow|L|$, where $K$ and $L$ are finite simplicial complexes with geometric realizations $|K|$ and $|L|$, is simplicial when it carries simplexes linearly into simplexes and is $P L$ when there are

Received by the editors February 19, 1971 and, in revised form, March 1, 1972.

AMS 1970 subject classifications. Primary 05C10; Secondary 55A15.

Key words and phrases. Crossing number, genus, immersion, square immersion, cube, cogenus.

(c) American Mathematical Society 1972 
subdivisions $K^{\prime}$ and $L^{\prime}$ of $K$ and $L$, respectively, with respect to which $f$ is simplicial. One can extend this definition to maps between triangulable spaces with respect to compatible families of triangulations (see Zeeman [4]) called PL structures. But any two PL structures on a graph or a closed 2-manifold are PL-equivalent (i.e. the Hauptvermutung holds for graphs and closed 2-manifolds), and so we can and will require immersions to be PL without specifying PL structures on our graphs or manifolds.

In this paper, we could easily get along without the PL assumptions and, in fact, the reader who desires to may simply omit them. However, since it is often convenient to know that an immersion can not be too "bad", we felt that spelling out the justification here would be useful as a future reference.

If $\Sigma_{g}$ and $\Sigma_{h}$ are surfaces of genus $g$ and genus $h$, respectively, their connected sum is obtained by cutting out discs from each surface and joining the discs by a hollow tube. The genus of the resulting surfaces is $g+h$. If we join $\Sigma_{g}$ and $\Sigma_{h}$ by $m$ tubes, $m \geqq 1$, then the new surface $\Sigma_{k}$ consists of $\Sigma_{g+h}$ with $m-1$ new handles; i.e., $k=g+h+m-1$.

Finally, if $G$ is a graph, the genus of $G, \gamma(G)$, is the smallest nonnegative integer $g$ such that there exists an imbedding of $G$ in $\Sigma_{g}$. This agrees with the usual (non-PL) notion of genus since any imbedding can be approximated arbitrarily closely by a PL imbedding.

Let $Q(d)$ denote the graph whose vertices are functions $f:\{1,2, \cdots, d\} \rightarrow$ $\{0,1\}, d \geqq 0$, where two functions $f$ and $g$ are considered adjacent if $f(i) \neq$ $g(i)$ for precisely one value of $i \in\{1,2, \cdots, d\}$. More geometrically $Q(d)$ is just the 1-skeleton (vertices and edges) of a $d$-dimensional cube. Let $\gamma=\gamma(Q(d))$.

The chief result of this paper is that the minimum number of edge crossings, $\mathrm{cr}_{\gamma-k}(Q(d))$, with which $Q(d)$ can be immersed in a surface of genus $\gamma-k(0 \leqq k \leqq \gamma)$, satisfies the inequalities

$$
4 k \leqq \mathrm{cr}_{\gamma-k}(Q(d)) \leqq 8 k
$$

provided that $k$ is sufficiently "small" compared to $d$-specifically, $k \leqq 2^{d-4}$. We conjecture that the second inequality in $\left({ }^{*}\right)$ is an equality.

The outline of the paper is as follows: In $\S 1$ we describe how a cube may be regarded as a cube of cubes and we define the notion of a square immersion. In $\$ 2$ we prove Lemma 1 which allows us to put together two square immersions of $Q(d)$ to obtain a square immersion of $Q(d+1)$. Next, in $\S 3$, we use Lemma 1 to obtain the results of [1] and [3] on the genus of the cube. This occupies Corollaries 1 and 2. We also define the cogenus of an immersion and prove Lemma 2 which sharpens Lemma 1 by including cogenus information. After the trivial constructive Lemma 3, we proceed to $\S 4$ where we obtain, by an inductive application of Lemmas 
1 and 2, Theorem 1 which yields the second inequality of $\left({ }^{*}\right)$. The first inequality of $\left({ }^{*}\right)$ (without restriction on $k$ ) is given in Theorem 2 as a consequence of [2]. The main result is Corollary 3.

We wish to thank the referee for his helpful suggestions.

1. Cubes cubed. Let $d$ be a nonnegative integer. We denote by $Q(d)$ the graph which is the 1 -skeleton of a $d$-dimensional cube. If $d=d^{\prime}+d^{\prime \prime}$ and $d^{\prime}, d^{\prime \prime} \geqq 0$, then we may regard $Q(d)$ as a copy of $Q\left(d^{\prime}\right)$ with each vertex $v \in V\left(Q\left(d^{\prime}\right)\right)$ replaced by a copy $Q\left(d^{\prime \prime}\right)_{v}$ of $Q\left(d^{\prime \prime}\right)$ and each edge $e$ from $v$ to $w$ replaced by the set of edges

$$
Q\left(d^{\prime \prime}\right)_{e}=\left\{x_{c} \mid x_{e} \text { is an edge from } x_{v} \text { to } x_{w}\right\},
$$

where $x_{v}$ is the vertex of $Q\left(d^{\prime \prime}\right)_{v}$ corresponding to the vertex $x \in V\left(Q\left(d^{\prime \prime}\right)\right)$. We denote this by writing

$$
Q(d)=Q\left(d^{\prime}\right) Q\left(d^{\prime \prime}\right)
$$

and we refer to (2) as a decomposition of $Q(d)$. For example, $Q(3)=$ $Q(2) Q(1)=Q(1) Q(2)$.

Note that decompositions are not unique. For example, we may regard a square as a line of lines in two different ways. Decomposition is also called product and Cartesian product.

Suppose $\varphi: Q(d) \rightarrow \Sigma_{g}$ is an immersion and $Q(d)=Q\left(d^{\prime}\right) Q\left(d^{\prime \prime}\right)$. Then we obtain, for each $v \in V\left(Q\left(d^{\prime}\right)\right)$, an immersion $\varphi_{v}: Q\left(d^{\prime \prime}\right)_{v} \rightarrow \Sigma_{g}$. Now suppose that $d \geqq 2$ and that $\varphi: Q(d) \rightarrow \Sigma_{g}$ is an immersion such that for some decomposition $Q(d)=Q(d-2) Q(2)$, each induced immersion $\varphi_{v}: Q(2)_{v} \rightarrow \Sigma_{g}$ is an imbedding with image bounding a closed disc $D_{v}$ with $D_{v} \cap \operatorname{im}(\varphi)=$ $\operatorname{im} \varphi_{r}$. Then we call $\varphi$ a square immersion. Thus, an immersion of $Q(d)$ is square if we can regard its image as a $d-2$-cube of squares each bounding a disc.

Let $G$ be a graph and suppose $\varphi: G \rightarrow \Sigma_{g}$ is an immersion. Then the crossing number, $\operatorname{cr} \varphi$, of $\varphi$ is the number of points $a$ in the image of $\varphi$ with

$$
a \in \varphi\left(\operatorname{int}\left(e_{1}\right)\right) \cap \varphi\left(\operatorname{int}\left(e_{2}\right)\right)
$$

for edges $e_{1} \neq e_{2} \in E(G)$, where int $(e)$ denotes the interior of $e$ for $e \in E(G)$. Call $\varphi: G \rightarrow \Sigma_{g}$ minimal if $\psi: G \rightarrow \Sigma_{g}$ implies $\operatorname{cr} \psi \geqq \operatorname{cr} \varphi$.

Our assumption that all maps are PL insures $\operatorname{cr} \varphi<\infty$ for all $\varphi$; but clearly $\operatorname{cr} \varphi<\infty$ when $\varphi$ is minimal, and it is with minimal immersions with which we shall be primarily concerned. We note that if $\varphi$ is minimal, then for any point $a$ as in (3) we must have the endpoints of $e_{1}$ disjoint from the endpoints of $e_{2}$. Moreover, given $a, b \in \varphi\left(\operatorname{int}\left(e_{1}\right)\right) \cap \varphi\left(\operatorname{int}\left(e_{2}\right)\right)$, we have $a=b$. Thus, in a minimal immersion, no edge meets itself and two distinct edges intersect in at most one common point. 
2. Building square immersions. Given two square immersions $\varphi_{1}$ and $\varphi_{2}$ of $Q(d)$ in $\Sigma_{g_{1}}$ and $\Sigma_{g_{2}}$, respectively, we can build a square immersion $\varphi$ of $Q(d+1)$ by connecting corresponding squares with tubes and running four edges along each tube. More precisely, we have the following lemma which will be our chief tool in the proof of the main theorem.

LEMMA 1. Let $d \geqq 2$ and let $\varphi_{1}: Q(d) \rightarrow \Sigma_{g_{1}}$ and $\varphi_{2}: Q(d) \rightarrow \Sigma_{g_{2}}$ be square immersions. Let $c_{j}=\operatorname{cr} \varphi_{j}, j=1,2$. Then there is a square immersion $\varphi: Q(d+1) \rightarrow \Sigma_{g}$ satisfying:

(a) $g=g_{1}+g_{2}+2^{d-1}-1$;

(b) $\operatorname{cr} \varphi=c_{1}+c_{2}$;

(c) given the decomposition $Q(d+1)=Q(1) Q(d)$ and denoting the vertices of $Q(1)$ by $v$ and $w$, we have $\varphi_{v}=\varphi_{1}$ and $\varphi_{w}=\varphi_{2}$.

Proof. Consider the decompositions $Q(d)=Q(d-2) Q(2)$ such that for every $y \in V(Q(d-2))$ we have induced imbeddings $\varphi_{1 y}: Q(2)_{y} \rightarrow \Sigma_{g_{1}}$ and $\varphi_{2 y}: Q(2)_{y} \rightarrow \Sigma_{g_{2}}$ whose images bound discs $D_{1 y}$ and $D_{2 y}$, respectively. Now, for each $y \in V(Q(d-2))$, connect the discs $D_{1 y}$ and $D_{2 y}$ by a (hollow) tube $T_{y}$ and call the resulting surface $\Sigma_{g}$. We shall determine $g$ in a moment.

First let us see how to define an immersion

$$
\varphi: Q(d+1)=Q(1) Q(d) \rightarrow \Sigma_{g}
$$

Let $\varphi \mid Q(d)_{v}=\varphi_{1}$ and $\varphi \mid Q(d)_{w}=\varphi_{2}$. Suppose $e$ denotes the only edge of $Q(1)$. We must now extend $\varphi$ over the set $Q(d)_{e}$ of edge-joining corresponding vertices in $Q(d)_{v}$ and $Q(d)_{w}$. But $Q(d)=Q(d-2) Q(2)$ so we can express $Q(d)_{e}$ as a disjoint union

$$
Q(d)_{e}=\bigcup_{y \in V(Q(d-2))} Q(2)_{e v}
$$

where $Q(2)_{e y}$ is the set of four edges joining vertices in $Q(2)_{v y}$ to corresponding vertices in $Q(2)_{w y}$.

Thus, it suffices to extend $\varphi$ over each $Q(2)_{e y}$. But $\varphi \mid Q(2)_{v y}=\varphi_{1 y}$ and $\varphi \mid Q(2)_{w y}=\varphi_{2 y}$. Hence, choosing four noninteresting rectilinear paths (along $T_{y}$ ) between the corresponding pairs of vertices in $Q(2)_{v y}$ and $Q(2)_{w y}$, we may obviously PL extend $\varphi$ over the edges in $Q(2)_{e y}$.

Attaching the tubes $T_{y}$ has destroyed the square immersion properties of $\varphi_{1}$ and $\varphi_{2}$ with respect to the squares $Q(2)_{v y}$ and $Q(2)_{w y}$. But if we take corresponding sides of the squares $Q(2)_{v y}$ and $Q(2)_{w y}$, then the two rectilinear paths along $T_{y}$ provide us with a new square imbedded in $\Sigma_{g}$ in the appropriate fashion. Thus, $\varphi$ is a square immersion.

Now we want to compute $g$. Since we have added one tube $T_{y}$ for each vertex $y \in V(Q(d-2))$, we have

$$
g=g_{1}+g_{2}+2^{d-2}-1 \text {. }
$$


Moreover, $\operatorname{cr} \varphi=\operatorname{cr} \varphi_{1}+\operatorname{cr} \varphi_{2}$ since $\varphi \mid Q(d)_{e}$ is an imbedding and $\varphi \mid Q(d)_{v}=\varphi_{1}$ and $\varphi \mid Q(d)_{w}=\varphi_{2}$. But now we have shown that $\varphi$ satisfies (a), (b) and (c).

3. Genus and cogenus. We can use Lemma 1 to rederive the result of Beineke and Harary [1] and Ringel [3] on the genus of $Q(d)$.

Corollary 1. Let $d \geqq 2$. Then $\gamma(Q(d)) \leqq 2^{d-3}(d-4)+1$. In particular. there is a square imbedding of $Q(d)$ in $\Sigma_{g}$ where $g=2^{d-3}(d-4)+1$.

Proof. We induct on $d$. When $d=2$, the result is clear. Now suppose the result holds for $d \geqq 2$. We prove it then holds for $d+1$. Consider the decomposition $Q(d+1)=Q(1) Q(d)$. By the inductive hypothesis there exist $\varphi_{j}: Q(d) \rightarrow \Sigma_{g_{j}}$ square imbeddings with $g_{j}=2^{d-3}(d-4)+1 \quad(j=1,2)$. Applying Lemma 1 , we obtain a square immersion $\varphi: Q(d+1) \rightarrow \Sigma_{g}$ with $g=g_{1}+g_{2}-2^{d-2}+1=2^{d-2}(d-3)+1$. Moreover, $\operatorname{cr} \varphi=0$ so $\varphi$ is an imbedding.

COROLlaRY 2. Let $d \geqq 2$. Then $\gamma(Q(d))=2^{d-3}(d-4)+1$.

ProOf. The reverse inequality to the preceding corollary is a direct consequence of the usual argument using Euler's formula. Alternatively, we could appeal to Theorem 2 of the sequel.

Let $G$ be a graph of genus $\gamma(G)$. Suppose $\varphi: G \rightarrow \Sigma_{g}$ is an immersion with $g \leqq \gamma(G)$. Let $k=\gamma(G)-g$. Then we say that $\varphi$ is an immersion of cogenus $k$ and write $\operatorname{cog} \varphi=k$.

LEMMA 2. Let $\varphi_{j}: Q(d) \rightarrow \Sigma_{g_{j}}$ be square immersions with cogenus $\varphi_{j}=k_{j}$ $(j=1,2)$. Then the square immersion $\varphi: Q(d+1) \rightarrow \Sigma_{g}$ constructed in Lemma 1 has $\operatorname{cog} \varphi=k_{1}+k_{2}$.

Proof. By Lemma 1, we have $g=g_{1}+g_{2}+2^{d-2}-1$. But $g_{j}=2^{d-3}(d-4)+$ $1-k_{j}(j=1,2)$ and so

$$
\begin{aligned}
\operatorname{cog} \varphi= & 2^{d-2}(d-3)+1-2^{d-3}(d-4)-1 \\
& +k_{1}-2^{d-3}(d-4)-1+k_{2}-2^{d-2}+1=k_{1}+k_{2} .
\end{aligned}
$$

Now we shall need one constructive lemma to establish the basis case of a later induction.

LEMMA 3. There exists a square immersion $\zeta: Q(4) \rightarrow \Sigma_{0}$ with $\operatorname{cog} \zeta=1$, cr $\zeta=8$. 
Proof. The following drawing shows how to immerse $Q(4)$ in $\Sigma_{0}$ :

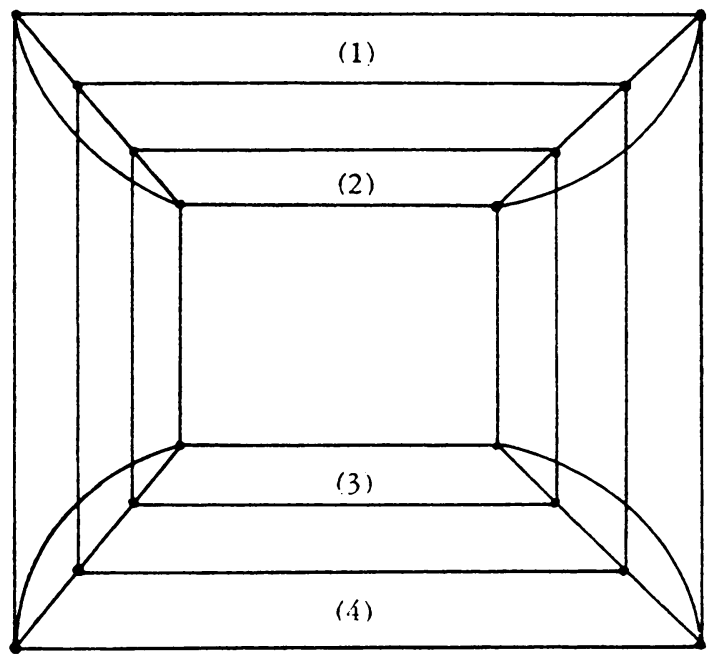

The reader may easily verify that $Q(4)$ is thus a square of squares with "vertices" (1), (2), (3), (4). If we let $\zeta$ be the corresponding square immersion whose image is the drawing above, clearly $\operatorname{cr} \zeta=8$ and $\operatorname{cog} \zeta=1$ since $\gamma(Q(4))=1$.

Note that $\zeta$, qua map, is not uniquely determined by the drawing since we can vary the parametrization along the edges. Moreover, we can also stretch and bend the image of $\zeta$. But we only care that there exist some map $\zeta$. One may clearly take $\zeta$ to be PL.

4. Stable crossing numbers. If $G$ is a graph and $\Sigma_{g}$ is a surface, we define $\operatorname{cr}_{g}(G)=\operatorname{cr} \varphi$, where $\varphi: G \rightarrow \Sigma_{g}$ is any minimal immersion. Obviously then $\gamma=$ genus of $G$ if and only if $\operatorname{cr}_{\gamma}(G)=0$ and $0 \leqq r<\gamma$ implies $\operatorname{cr}_{r}(G)>0$. Now suppose we consider $r=\gamma-k, k \geqq 0$. We write $\operatorname{cr}^{k}(G)$ instead of $\mathrm{cr}_{y-k}(G)$. Thus $\operatorname{cr}^{k}(G)=\mathrm{cr} \varphi$, where $\varphi$ is a minimal immersion of cogenus $k$.

Suppose we consider the case $G=Q(d)$ for some $d \geqq 0$. Clearly, $\operatorname{cr}^{0}(Q(d))=$ 0 for all $d$-in fact, $\operatorname{cr}^{0}(G)=0$ for any graph. We proved in an earlier paper [2] that, for $d \geqq 4$,

$$
4 \leqq \operatorname{cr}^{1}(Q(d)) \leqq 8 .
$$

The following two theorems show that the above situation is quite general, provided only that $k$ is sufficiently small compared to $d$. Because of its formal similarity to stable-range imbedding and immersion problems for manifolds, we call such a phenomenon stable.

THEOREM 1. Suppose $d \geqq 4$ and $0 \leqq k \leqq 2^{d-4}$. Then $\operatorname{cr}^{k}(Q(d)) \leqq 8 k$. In fact, there is a square immersion $\varphi$ of cogenus $k$ with $\operatorname{cr} \varphi=8 k$. 
Proof. We induct on $d$. First suppose $d=4$. If $k=0$, the result holds by Corollary 1 . If $k=1$, the result holds by Lemma 3 .

Suppose the result holds for some $d \geqq 4$. We prove it holds for $d+1$. Suppose $0 \leqq k \leqq 2^{d-3}$. Now choose $k_{1}, k_{2}$ so that $k=k_{1}+k_{2}$ and $k_{1}, k_{2} \leqq$ $2^{d-4}$. Then, by the induction hypothesis, there exist square immersions

$$
\varphi_{j}: Q(d) \rightarrow \Sigma_{g_{j}}
$$

with $\operatorname{cog} \varphi_{j}=k_{j}, \quad \operatorname{cr} \varphi_{j}=8 k_{j} \quad(j=1,2)$. Consider the decomposition $Q(d+1)=Q(1) Q(d)$. Then by Lemmas 1 and 2 there is a square immersion $\varphi: Q(d+1) \rightarrow \Sigma_{g}$ with $\operatorname{cog} \varphi=k_{1}+k_{2}=k$ and

$$
\operatorname{cr} \varphi=\operatorname{cr} \varphi_{1}+\operatorname{cr} \varphi_{2}=8 k_{1}+8 k_{2}=8 k \text {. }
$$

This completes the proof.

Let us recall from [2] that, for $d \geqq 2$ and $g \geqq 0$,

$$
\mathrm{cr}_{g}(Q(d)) \geqq \delta_{g}(Q(d)),
$$

where

$$
\delta_{g}(Q(d))=d 2^{d-1}-2\left(2^{d}-2(1-g)\right) .
$$

Now suppose $k \geqq 0$ and $g=\gamma(Q(d))-k$. Then

$$
\begin{aligned}
\delta_{g}(Q(d)) & =d 2^{d-1}-2^{d+1}+4-4 \gamma(Q(d))+4 k \\
& =d 2^{d-1}-2^{d+1}+4-2^{d-1}(d-4)-4+4 k=4 k,
\end{aligned}
$$

and, therefore, since $\mathrm{cr}_{y-k}(Q(d))=\mathrm{cr}^{k}(Q(d))$, we have

$$
\operatorname{cr}^{k}(Q(d)) \geqq 4 k
$$

for $d \geqq 2$. (Of course, the result also holds for $d=0,1$.) Therefore, we have the following theorem.

THEOREM 2. Let $0 \leqq k \leqq \gamma(Q(d))$ for $d \geqq 0$. Then $\operatorname{cr}^{k}(Q(d)) \geqq 4 k$.

In the stable range of $d \geqq 4$ and $0 \leqq k \leqq 2^{d-4}$, we can put together Theorems 1 and 2 to obtain the following:

COROLlaRY 3. Suppose $d \geqq 4$ and $0 \leqq k \leqq 2^{d-4}$. Then

$$
4 k \leqq \operatorname{cr}^{k}(Q(d)) \leqq 8 k .
$$

We conjecture that, in fact, the second inequality is an equality. In particular, we conjecture that for fixed $k, \mathrm{cr}^{k}(Q(d))$ is independent of $d$. If this latter conjecture is true, then $\mathrm{cr}^{k}(Q(d))$ would qualify as the stable crossing number of cubes in cogenus $k$. What we have actually shown is that stably $4 k \leqq \mathrm{cr}^{k}(Q(d)) \leqq 8 k$. 


\section{REFERENCES}

1. L. W. Beineke and F. Harary, The genus of the n-cube, Canad. J. Math. 17 (1965), 494-496. MR 31 \#81.

2. P. C. Kainen, A lower bound for crossing numbers of graphs with applications to $K_{n}, K_{p, q}$, and $Q(d)$, J. Combinatorial Theory Ser. B 12 (1972), 287-298.

3. G. Ringel, Über drei kombinatorische Probleme am n-dimensionalen Würfel und Würfelgitter, Abh. Math. Sem. Univ. Hamburg 20 (1955), 10-19. MR 17, 772.

4. E. C. Zeeman, Seminar on combinatorial topology, Chap. 2, Institut des Hautes Etudes Scientifiques, 1963 (mimeographed).

Department of Mathematics, Case Western Reserve University, Cleveland, ОHIO 44106 